\title{
Evaluation of Distensibility and Stiffness of Ascending Aortic Aneurysm using Magnetic Resonance Imaging
}

\author{
Kaushal Kishore Tiwari, ${ }^{1,2}$ Stefano Bevilacqua, ${ }^{2}$ Giovanni Aquaro, ${ }^{3}$ Pierluigi Festa, ${ }^{3}$ Lamia Ait-Ali, ${ }^{3}$ Marco Solinas ${ }^{2}$ \\ 'Department of Cardiothoracic and Vascular Surgery, College of Medical Sciences, Teaching Hospital, Bharatpur, Chitwan, \\ Nepal, ${ }^{2}$ Department of Adult Cardiac Surgery, FTGM, G. Pasquinucci Heart Hospital, Massa, Italy, ${ }^{3}$ MRI Laboratory, FTGM, \\ CNR, Pisa, Italy.
}

Introduction: Magnetic resonance imaging emerging as a new tool for the diagnosis and evaluation of ascending aortic aneurysm. The aim of our study is to evaluate in vivo distensibility and pulse wave velocity of the aortic wall using functional magnetic resonance imaging technique.

Methods: We enrolled 25 patients undergoing surgery for ascending aortic aneurysm and or aortic valve replacement for a period of 8 months. Preoperatively, all the patients underwent functional MRI study of the aorta. Aortic wall distensibility and pulse wave velocity of ascending aorta was evaluated.

Results: Mean age of the patient was 66 years (66.68 \pm 5.62 years) with $60 \%$ (15) male patients. More than fifty percentages of patients were smoker (52\%), hypertensive $(64 \%)$ and diabetic (56\%). We have observed significant decrease of distensibilty in the patients with aortic diameter above $50 \mathrm{~mm}$ ( $\mathrm{p}-0.0002)$. Furthermore, we have found a significant inverse correlation between aortic distensibility and pulse wave velocity $\left(R=-0.650, R^{2}=0.42, p-0.0004\right)$. Similarly, we have found a significant inverse correlation between ascending aortic diameter and distensibility of the aorta $\left(R=-0.785, R^{2}=0.61\right.$, $\mathrm{p}-0.00001)$. Statistically significant positive correlation was observed between aortic diameter and pulse wave velocity $\left(R=0.865, R^{2}=0.74, p-0.00001\right)$.

Conclusions: MRI measurement of aortic diameters, distensibility, and flow wave velocity is an easy, reliable and reproducible technique. Distensibility and pulse wave velocity define the elasticity of the aorta. We have observed that elasticity of aortic wall is decreased in ascending aorta aneurysm patients.

Keywords: ascending aorta aneurysm; distensibility; pulse wave velocity; MRI.

\section{INTRODUCTION}

MRI has shown high sensibility and specificity both for initial diagnosis and progression of aneurismal disease. Most of the study to evaluate ascending aorta using functional MRI has been done in Marfan patients or patients with bicuspid aortic valve. ${ }^{1}$ However, its role in evaluating biomechanical property of the ascending aorta in other patients could be valuable as well. Information supplied by functional MRI may have utility in thoracic aortic disease management.

Correspondence: Dr. Kaushal K Tiwari, Department of Cardiothoracic and Vascular Surgery, College of Medical Sciences Teaching Hospital, Bharatpur, Chitwan, Nepal. Email: drkaushalkt@ yahoo.com, Phone: $+977-9801059361$. 
Important parameters evaluated by functional MRI are distensibility, pulse wave velocity and maximal rate of systolic distension and maximal rate of diastolic recoil. ${ }^{2}$

Aim of our study is to evaluate in vivo distensibility and pulse wave velocity, defining stiffness of the aortic wall using functional magnetic resonance imaging (MRI) technique.

\section{METHODS}

We have selectively enrolled 25 patients undergoing surgery for ascending aortic aneurysm and or aortic valve replacement in the department of adult cardiac surgery, G. Paquinucci Heart Hospital, Massa, Italy for a period of 8 months. Written consent from patients has taken and institutional ethical committee has approved the study protocol. Patients with known genetic disorders associated to specific aortic wall alterations (Marfan syndrome, Ehlers-Danlos syndrome), autoimmune chronic inflammatory diseases (Sjögren disease, Systemic Lupus Erythematosus, etc), chronic renal and hepatic failure, infectious aneurysm (syphilis, fungal, etc), active cancer or immunoproliferative diseases (leukemia, lymphomas etc), Re-do open heart procedures and patients with contraindications for MRI were excluded from the study. Preoperatively, all the patients underwent functional MRI study of the aorta by a 1.5 Tesla scanner (Signa Hdx, General Electrics Healthcare, WI, USA) with eight-channel cardiac phaged array coil. The thoracic aorta was scanned by acquiring sagittal-oblique cine images parallel to the major aortic axis using a breath hold, ECG-triggered, steady-state fast precession (SSFP) pulse sequence with the following parameters: $400 \mathrm{~mm}$ field of view, 8-mm slice thickness, no gap, 1 EX, 12 views per segment. The number of cardiac phases set according to the heart rate to obtain an aortic wall excursion temporal resolution of about $10 .^{3}$

Cross-sectional cine SSFP images with the same parameters were acquired at different aortic levels: 1) at the aortic valve plane to evaluate the aortic valve morphology and to quantify the planimetric area; 2) at the aortic root; 3) at the sinotubular junction; 4) at the proximal ascending aorta $(5 \mathrm{~mm}$ above the sinotubular junction); and 5) at the level of maximum diameter of the ascending aorta.

A commercially available software program (Mass Plus and CV flow, Leiden, The Netherlands) was used for the initial image acquisition and processing. Furthermore, aortic contours were drawn manually and then processing of these images, to calculate these new indexes, was done using a dedicated computer program, specifically developed at FTGM, MRI laboratory, Pisa

(Figure 1).
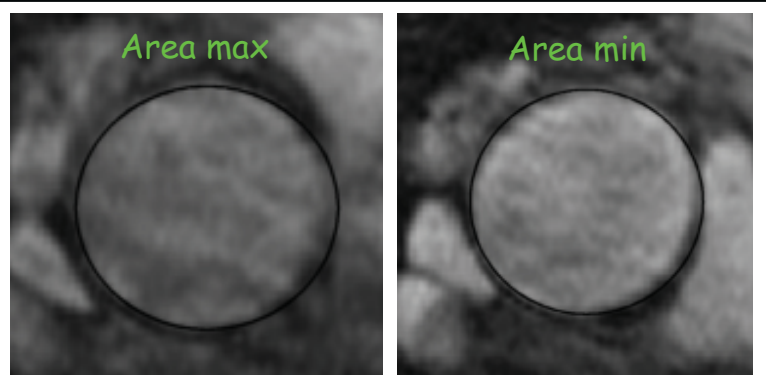

Figure 1. Acquisition and processing of the MRI images.

Aortic wall distensibility (D) of ascending aorta was evaluated in SSFP images using follow formula suggested by Grotenhius et al. $^{3}$ $D=(A \max -A \min ) /[A \min x(P \max -P \min )]$

Where A max is the maximal (systolic) aortic area $\left(\mathrm{mm}^{2}\right), A$ min was the minimal (diastolic) aortic area (in $\mathrm{mm}^{2}$ ), $\mathrm{P}$ max was the systolic blood pressure (in $\mathrm{mmHg}$ ), and $\mathrm{P}$ min the diastolic blood pressure (in $\mathrm{mmHg}$ ). Distensibility was expressed as $10^{-3} / \mathrm{mmHg}$.

Flow wave velocity (FWV) was calculated as the ratio of the distance between two levels and the time difference between arrivals of the flow wave at these levels.

It is calculated by means of the following formula: FWV - Distance Ascending Aorta - Distance Descending Aorta/ Tdelay It is expressed as meter per sec $(\mathrm{m} / \mathrm{s})$ (Figure 2).

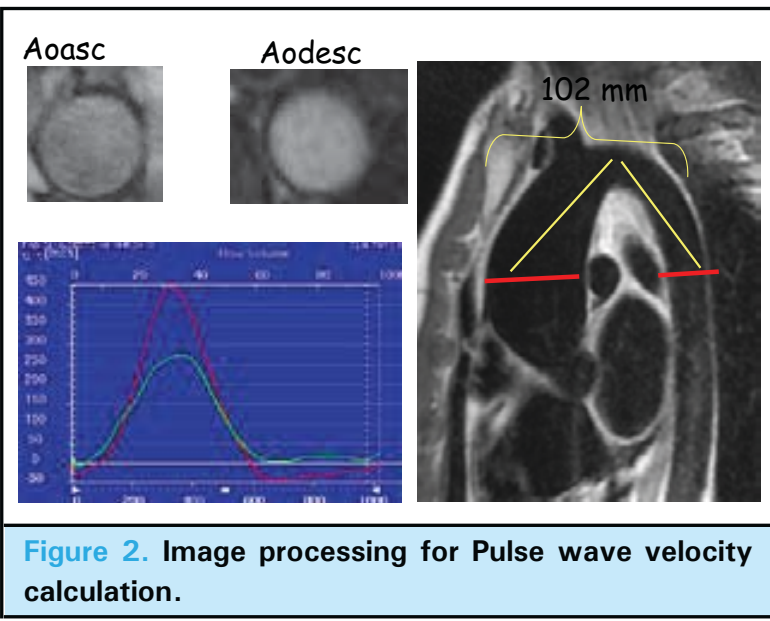

Where $T_{\text {delay }}$ is the time delay of the flow velocity peak between descending and ascending aorta and $D_{\text {Asc-Desc }}$ was the distance between ascending and descending aorta along the aortic vessel measured. 
Statistical analysis: All the statistical evaluation were done using STATVIEW 5.0 version software (SAS Institute, Cary, NC, USA). Group data were summarized by mean and standard deviation or by frequency percentages. Pearson correlation coefficient calculated and the relationships between variables were evaluated by single and multiple linear regression analysis. Statistical value with $\mathrm{p}<0.05$ considered statistically significant.

\section{RESULTS}

Baseline clinical characteristics of the patient shown in the Table 1. Mean age of the patient was 66 years (66.68 \pm 5.62 years) with $60 \%$ (15) male patients. More than fifty percentages of patients were smoker $(52 \%)$, hypertensive (64\%) and diabetic (56\%). Mean aortic root and ascending aortic diameter were $40.12 \pm$ 3.32 and $52.8 \pm 4.26$ respectively. Five patients were diagnosed with bicuspid aortic valve, whereas three patients with aortic dissection. Overall distensibility was decreased in these patient when taken as mean $(2,68$ $\pm 0,34)$. Similarly pulse wave velocity was increased in average $5,83 \pm 0,62$. While we divided these patients in two groups. Nine having aortic diameter up $50 \mathrm{~mm}$ and another 16 patients having aortic diameter above $50 \mathrm{~mm}$.

\begin{tabular}{|c|c|c|}
\hline SN & $\begin{array}{l}\text { Baseline Clinical } \\
\text { Characteristics }\end{array}$ & Values \\
\hline 1. & Age (Mean \pm SD) & $66.68 \pm 5.62$ \\
\hline 2. & Sex & $\begin{array}{l}\text { Male - } 15 \text { pts }(60 \%) \\
\text { Female }-10 \text { pts }(40 \%)\end{array}$ \\
\hline 3. & Smoker & 13 pts $(52 \%)$ \\
\hline 4. & Diabetes & 14 pts $(56 \%)$ \\
\hline 5. & Hypertension & 16 pts $(64 \%)$ \\
\hline 6. & Dyslipidemia & 10 pts $(40 \%)$ \\
\hline 7. & $\begin{array}{l}\text { Aortic root diameter } \\
\text { (Mean } \pm \mathrm{SD} \text { ) }\end{array}$ & $40.12 \pm 3.32$ \\
\hline 8. & $\begin{array}{l}\text { Ascending aorta } \\
\text { diameter (Mean } \pm \\
\text { SD) }\end{array}$ & $52.8 \pm 4.26$ \\
\hline 9. & $\begin{array}{l}\text { Descending aorta } \\
\text { diameter (Mean } \pm \\
\text { SD) }\end{array}$ & $27.52 \pm 2.46$ \\
\hline 10. & Bicuspid Aortic valve & 5 pts $(20 \%)$ \\
\hline 11. & Dissection & 3 pts $(12 \%)$ \\
\hline 12. & $\begin{array}{l}\text { Distensibility }\left(10^{-3} /\right. \\
\mathrm{mmHg})\end{array}$ & $2,68 \pm 0,34$ \\
\hline 13. & $\begin{array}{l}\text { Pulse wave velocity } \\
\text { (m/s) }\end{array}$ & $5,83 \pm 0,62$ \\
\hline
\end{tabular}

We have observed significant decrease of distensibilty in the group with aortic diameter above $50 \mathrm{~mm} \mathrm{(p-}$ 0.0002) (Figure 3). Furthermore, we have found a significant inverse correlation between aortic distensibility and pulse wave velocity $\left(R=-0.650, R^{2}=\right.$ $0.42, p-0.0004$ ) (Figure 4). Similarly, we have found a significant inverse correlation between ascending aortic diameter and distensibility of the aorta $\left(R=-0.785, R^{2}=\right.$ $0.61, p-0.00001$ ) (Figure 5). Moreover, a statistically significant positive correlation was observed between aortic diameter and pulse wave velocity $(R=0.865$, $R^{2}=0.74, p-0.00001$ ) (Figure 6).
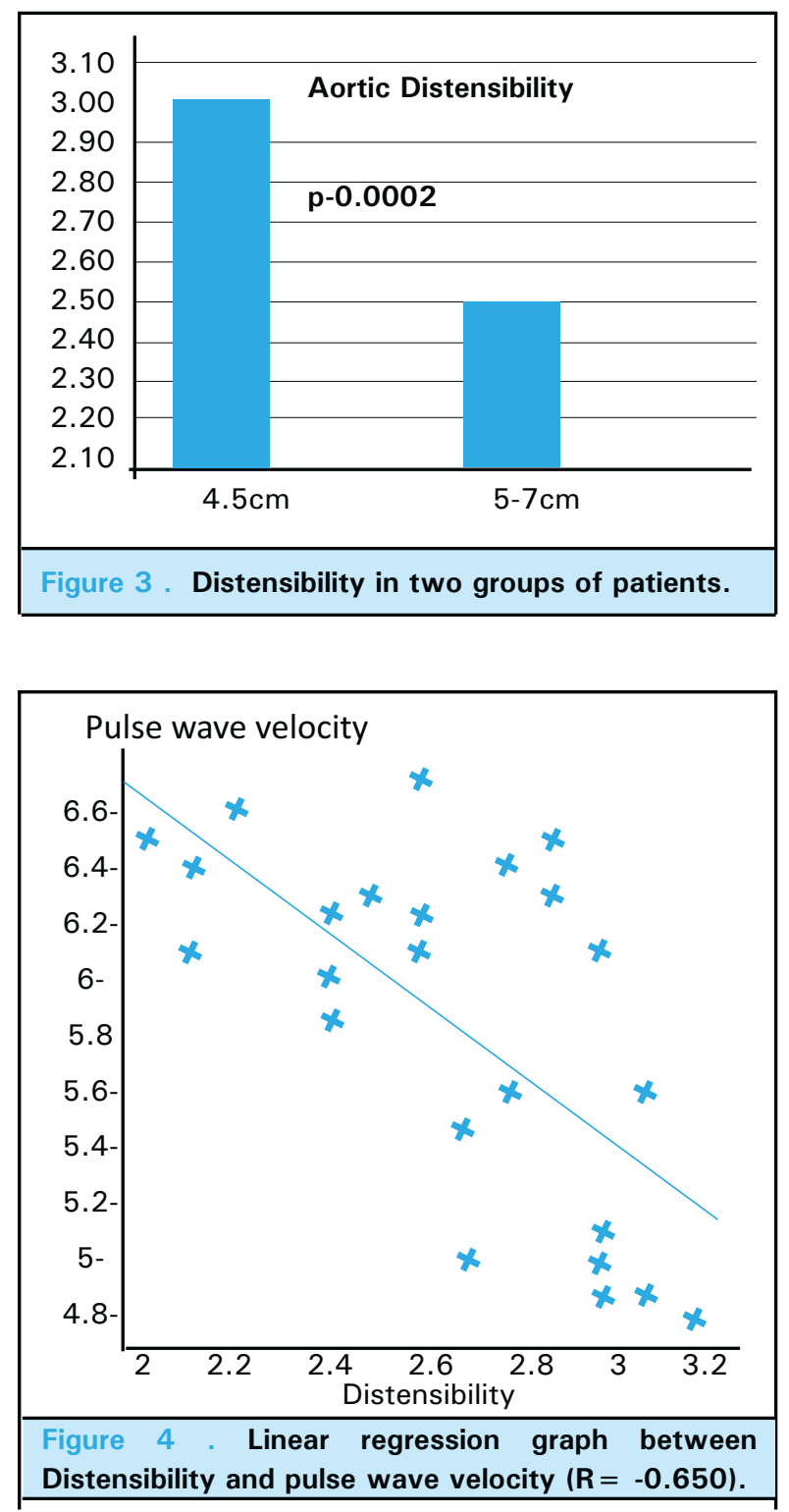
Tiwari et al. Evaluation of Distensibility and Stiffness of Ascending Aortic Aneurysm using Magnetic Resonance Imaging
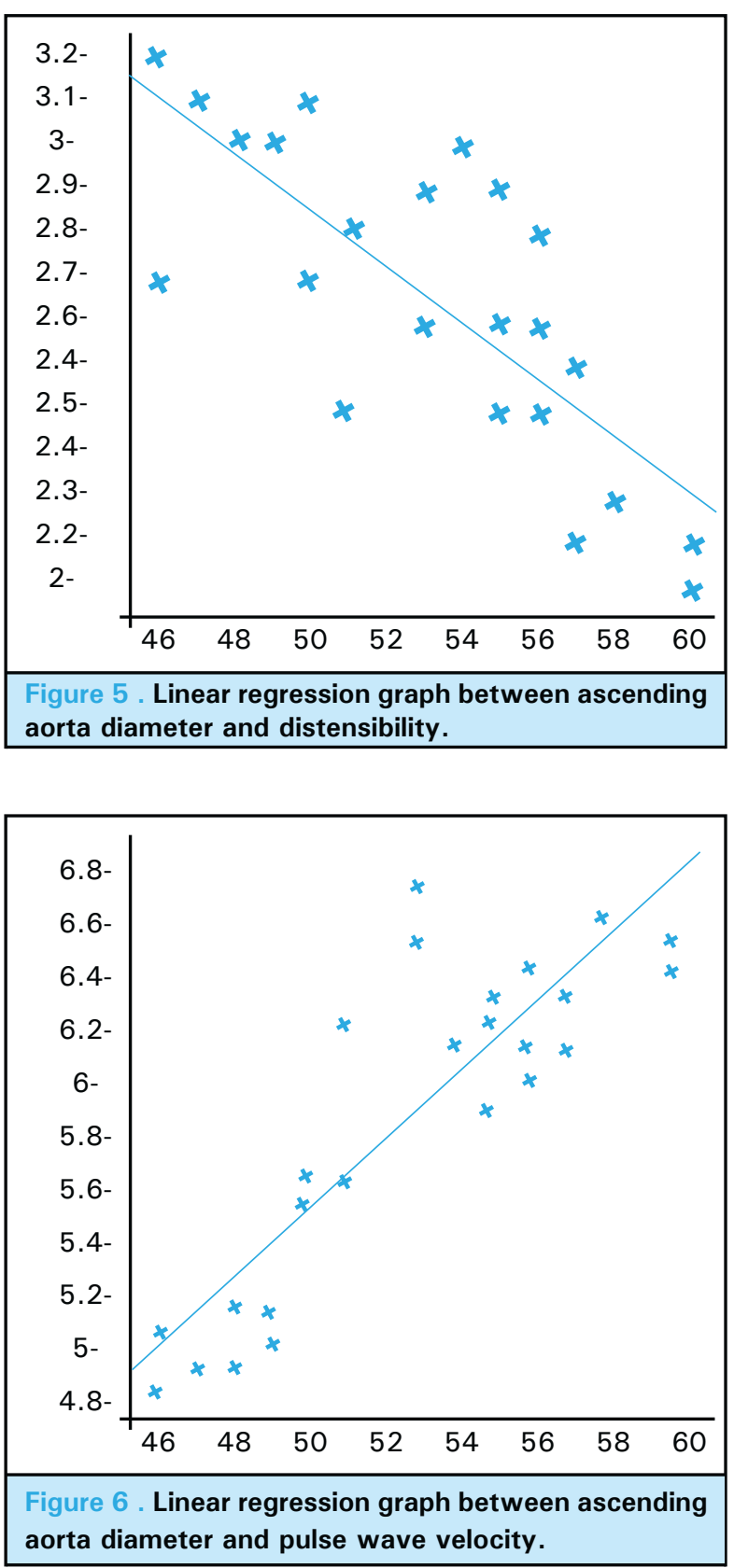

\section{DISCUSSION}

Thoracic aortic aneurysm (TAA) has a reported incidence of about 10 cases per 100000 patients/ year, with a yearly risk of rupture or dissection of $7 \% .^{5}$ Significant heterogeneity occurs in distribution of aneurysm disease along the aorta, with $50 \%$ of all TAA involving the ascending aorta, $10 \%$ the arch, and $40 \%$ the descending aorta. ${ }^{6}$

Currently, surgical treatment of TAA has indicated based on the diameter and growth rate of the aorta. Prophylactic surgery has advised for an aorta of 55 $\mathrm{mm}$ in normal subject, while $50 \mathrm{~mm}$ in patients with Marfan syndrome. ${ }^{5}$ However, most of the dissections or ruptures do not occur in patients falling within current guideline indication for elective surgery. IRAD data shows that more than $60 \%$ of the patients operated for aortic dissection had aortic diameter less than $55 \mathrm{~mm} .{ }^{7}$ These suggest that methods other than size measurement of the ascending aorta needed to identify patients at risk for dissection or rupture.

Assessment of ascending aortic elastic properties using MRI has been proposed in several studies. ${ }^{1,8}$ Aortic distensibility and pulse wave velocity as a parameter has used frequently to evaluate elastic property of the aorta. Most of the studies were performed in patients with Marfan syndrome or with bicuspid aortic valve. ${ }^{8,9}$ In our study, we have evaluated elastic property of the aorta using MRI by calculating distensibility and pulse wave velocity in patients with ascending aortic aneurysm without bicuspid valve and any genetic disorders. We have found that distensibility of the aorta decreases as the aorta enlarges. However, it still conserves the distensibility before it reaches a diameter of more than $50 \mathrm{~mm}$. Moreover, we have observed a negative correlation between aortic diameter and distensibility. Distensibility of the aorta significantly decreases in patients with aortic diameter more than 50 $\mathrm{mm}$ as well as when aortic diameter increases. This is an alarming condition. Patients with aortic aneurysm of more than $50 \mathrm{~mm}$ are at increased risk of dissection or rupture of the aorta because the aorta is stiff. Koullias et al, have shown that large aneurysms experience highly increased wall stress and in a physiologic range of blood pressure, they exhibit wall stress that equals or even exceeds the known ultimate tensile strength of the aortic tissue. ${ }^{8}$ These observations points towards a noteworthy mechanical deterioration of the elastic properties of these dilated aortas. Rupture of the aorta occurs when the stress exerted on the aortic wall exceed the aortic wall capacity to sustain stress during the cardiac cycle..$^{9,10}$ Furthermore, Iliopoulos et al have concluded that TAA development is not associated with mechanical weakening but with the stiffening and reduction in the vessel extensibility. ${ }^{12}$ Equally, Vorp et al have demonstrated, in a in vitro study, that TAA specimens are $30 \%$ weaker, but supported the fact that are stiffer than non-aneurismal ascending thoracic aorta. ${ }^{11}$ Therefore, aneurysmatic ascending aorta, which is stiff indeed, is prone to rupture due to its inability to cope with the pressure wave generated during each cardiac cycle.

The pulse wave velocity through the aorta is another measure for aortic stiffness. ${ }^{12}$ Additionally, we have found a significant negative and positive correlation between pulse wave velocity with aortic distensibility 
and aortic diameter, respectively. It means pulse wave velocity increases as the distensibility of the aorta decreases and size of the aorta increases, which implies a noncompliance aorta during the systolic stress. With increasing aortic diameter, the risk of aortic dissection increases, but dissection could also occur in non-dilated aortas. Consequently, aortic stiffness as a potential predictor of aortic dissection has been investigated in patients with Marfan syndrome. ${ }^{5}$ Several studies have demonstrated increased aortic stiffness assessed by echocardiography and $\mathrm{MRI}$ in patients with Marfan syndrome. 5,13,14 Ironically, the predictive value of aortic stiffness on progressive aortic dilatation has not been investigated well. As increased aortic stiffness precedes progressive aortic dilatation, therefore stiffness measurement could be a useful tool for the identification of patients at risk of dissection or rutpture.

It has been shown that both bicuspid aortic valve patients and Marfan patients had significantly faster flow propagation than controls, emphasizing that the ascending aorta in these patients are stiffer. The reduced aortic wall elasticity in bicuspid aortic valve patients could be the main cause of the increased flow velocity in the proximal thoracic aorta. ${ }^{15}$
Knowledge of the elastic property like distensibility, aortic wall strength, and of the forces acting on the aortic wall, could potentially provide diagnostic identification of those aneurysm with risk of rupture exceeding the risk of surgery. ${ }^{15} \mathrm{Up}$ to now, elastic property of the aorta has been evaluated mostly in ex vivo condition using surgical aortic wall specimens, ${ }^{16,17}$ but a in vivo evaluation of the elastic property by echocardiography or MRI gives clinicians an extra clue to decide the timing for surgical intervention.

\section{CONCLUSIONS}

Based on our findings, we conclude that elastic property of the aortic wall in ascending aortic aneurysm is decreased. MRI measurement of aortic diameters, distensibility, and flow wave velocity has been proven easy, available, affordable and reproducible. MRI could help surgeons in making proper and timely decision to intervene in patients with ascending aorta aneurysm to prevent fatal complications.

\section{Conflict of Interest: None.}

\section{REFERENCES}

1. Groenink M, de Roos A, Mulder BJ, Verbeeten B Jr, Timmermans J, ZwindermanAHet al. Biophysical properties of the normal-sized aorta in patients with Marfan syndrome: evaluation with MR flow mapping. Radiology 2001;219:535-40.

2. Aquaro GD, Cagnolo A, Tiwari KK, Todiere G, Bevilacqua S, Di Bella G, Ait-Ali L, Festa P, Glauber M, Lombardi M. Age-dependentchange in elasticproperty of thoracic aorta evaluated by magneticresonace in normalsubjects. Interactive Cardiovascular and Thoracic Surgery 2013; 17:674-79.

3. Grotenhuis HB, Ottenkamp J, Westenberg JJ, Bax JJ, Kroft LJ, de Roos A. Reduced aortic elasticity and dilatation are associated with aortic regurgitation and left ventricular hypertrophy in nonstenotic bicuspid aorticvalve patients. J Am CollCardiol2007;49:1660-5.

4. Nollen GJ, Groenink M, Tijssen JGP, var der Wall EE, Mulder BJM. Aortic stiffness and diameter predict progressive aortic dilatation in patients with Marfan Syndrome. European Heat Journal 2004;25:1146-52.

5. Elefteriades JA. Natural history of thoracic aortic aneurysms: indication for surgery, and surgical versus nonsurgical risks. Ann Thorac Surg 2002;74 (Suppl):1877-80.

6. Beckman JA. Aortic aneurysms: pathophysiology, epidemiology, and prognosis. In: Creager MA, Dzau VJ, Loscalzo J, eds. Vascular Medicine. 2006.

7. Hagan PG, Nienaber CA, Isselbacher EM, et al. The international registry of acute aortic dissection (IRAD): new insights into an old disease. JAMA 2000; 283(7):897-903.

8. Koullias G, Modak R, Tranquilli M, Korkolis DP, Barash P, Elefteriades JA. Mechanical deterioration underlies malignant behavior of aneurismal human ascending aorta. J ThoracCard iovascSurg2005;130:677-83
9. Davies RR, Goldstein LJ, Coady MA, Tittle SL, Rizzo JA, Kopf GS, Elefteriades JA. Yearly rupture or dissection reates for thoracic aortic aneurysm: simple prediction based on size. Ann ThoracSurg2002;73:17-27.

10. Iliopoulos DC, Kritharis EP, Giagini AT, Papadodima SA, Sokolis DP. Ascending thoracic aortic aneurysm are associated with compositional remodeling and vessel stiffening but not weakening in age matched subjects. J ThoracCardivascSurg2009;137:101-9.

11. Vorp DA, Schiro BJ, Ehrlich MP, Juvonen TS, Ergin MA, Griffith BP. Effect of aneurysm on the tensile strength and biomechanical behavior of the ascending thoracic aorta. Ann ThoracSurg 2003; 75:1210-4.

12. Bramwell JC, Hill AY. Velocity of transmission of the pulse-wave and elasticity of arteries. Lancet 1922; 891-2.

13. Aquaro GD, Ait-Ali L, Basso ML, Lombardi M, Pingitore A, Festa P. Elasticproperties of aortic wall in patients with bicuspid aortic valve by magneticresonance imaging. Am J Cardiol2011;108:81

14. Chen CH, Nevo E, Fetics B, Pak PH, Yin FC, Maughan WL et al. Estimationof central aortic pressure waveform by mathematical transformation ofradial tonometry pressure. Validation of generalized transfer function.Circulation 1997;95:1827-36

15. Raghavan ML, Vorp DA, Federle MP, Makaroun MS, Webster MW. Wall stress distribution on three-dimensionally reconstructed models of human abdominal aortic aneurysm- J VascSurg 2000;31:760-9.

16. Gracia-Herrara CM, Atienza JM, Rojo FJ, Claes E, Guinea GV, Celentano DJ, Garcia-Montero C, Burgos RL. Mechanical behavior and rupture of normal and pathological human ascending aortic wall. Med BiolEngComput2012;50:559-66.

17. Pasta S, Phillippi JA, Gleason TG, Vorp DA. Effect of aneurysm on the mechanical dissection properties of the human ascending thoracic aorta. J ThoracCardiovascSurg2012;143:460-67. 